\title{
On the Efficiency of Grain Alignment in Dark Clouds
}

\section{Citation}

Lazarian, A., Alyssa A. Goodman, and Philip C. Myers. 1997. "On the Efficiency of Grain Alignment in Dark Clouds." The Astrophysical Journal 490 (1): 273-80. https:// doi.org/10.1086/304874.

\section{Permanent link}

http://nrs.harvard.edu/urn-3:HUL.InstRepos:41397472

\section{Terms of Use}

This article was downloaded from Harvard University's DASH repository, and is made available under the terms and conditions applicable to Other Posted Material, as set forth at http:// nrs.harvard.edu/urn-3:HUL.InstRepos:dash.current.terms-of-use\#LAA

\section{Share Your Story}

The Harvard community has made this article openly available.

Please share how this access benefits you. Submit a story.

\section{Accessibility}


The Astrophysical JouRnal, 490:273-280, 1997 November 20

(C) 1997. The American Astronomical Society. All rights reserved. Printed in U.S.A.

\title{
ON THE EFFICIENCY OF GRAIN ALIGNMENT IN DARK CLOUDS ${ }^{1}$
}

\author{
A. Lazarian, ${ }^{2,3}$ Alyssa A. Goodman, ${ }^{4,5}$ AND Philip C. Myers ${ }^{3}$ \\ Received 1997 April 14; accepted 1997 July 2
}

\begin{abstract}
A quantitative analysis of grain alignment in the filamentary dark cloud L1755 in Ophiuchus is presented. We show that the observed decrease of the polarization-to-extinction ratio for the inner parts of this quiescent dark cloud can be explained as a result of the decrease of the efficiency of grain alignment. We make quantitative estimates of grain alignment efficiency for six mechanisms involving grains with either thermal or suprathermal rotation, interacting with either magnetic field or gaseous flow. We also make semiquantitative estimates of grain alignment by radiative torques. We show that in conditions typical of dark cloud interiors, all known major mechanisms of grain alignment fail. All the studied mechanisms predict polarization at least an order of magnitude below the currently detectable levels of $\sim 1 \%$. On the contrary, in the dark cloud environments where $A_{v}<1$, the grain alignment can be much more efficient. There the alignment of suprathermally rotating grains with superparamagnetic inclusions, and possibly also radiative torques, account for observed polarization. These results apply to L1755, which we model in detail, and probably also to B216 and other similar dark clouds. Our study suggests an explanation for the difference in results obtained through polarimetry of background starlight and polarized thermal emission from the dust itself. We conjecture that the emission polarimetry selectively reveals aligned grains in the environment far from thermodynamic equilibrium, as opposed to starlight polarization studies that probe the alignment of grains all the way along the line of sight, including the interiors of dark quiescent clouds, where no alignment is possible.
\end{abstract}

Subject headings: dust: extinction - ISM: clouds - polarization

\section{INTRODUCTION}

Discovered nearly half a century ago, alignment of interstellar dust (see Hiltner 1949; Hall 1949) has not yet been understood properly. Table 1 outlines some features of the major known mechanisms of grain alignment, which are discussed below. To find out when a particular mechanism is efficient is a challenging and exciting problem to be solved jointly by observers and theorists (see Hildebrand \& Dragovan 1995; Desch \& Roberge 1997). At present, the lack of a proper understanding of the alignment processes means that it is extremely difficult to disentangle variations in polarization efficiency from magnetic field variations in polarization maps.

The present research was initiated by recent studies of polarization of near-infrared background starlight seen through dark clouds, namely, B216-217 in Taurus (Goodman et al. 1992) and L1755 in Ophiuchus (Goodman et al. 1995). In both cases it was shown that the nearinfrared polarimetry of background starlight could not reveal magnetic field structure within these cold dense regions. In general, it seems that toward dark clouds polarization does increase with extinction for visual extinction $A_{v}<1$ but does not for $A_{v} \gg 1$ (Goodman 1996). A list of factors that can suppress polarization from cloud interiors was presented in Goodman et al. (1995). Here we analyze only one of those factors, namely, the difference in grain alignment. In other words, in our model we assume that

\footnotetext{
${ }^{1}$ We dedicate this paper to the memory of Edward M. Purcell and Lyman Spitzer, Jr., two pioneers in the quantitative study of the interstellar medium.

2 Princeton University Observatory, Princeton, NJ 08544.

${ }^{3}$ Harvard-Smithsonian Center for Astrophysics, 60 Garden Street, Cambridge, MA 02138.

${ }^{4}$ Department of Astronomy, Harvard University, 60 Garden Street, Cambridge, MA 02138.

${ }^{5}$ National Science Foundation Young Investigator.
}

grain shape, size, and composition, as well as magnetic field structure, are the same in the exterior and interior of molecular clouds. It is shown in Goodman (1996) that other factors in the aforementioned list can further decrease the efficiency of dichroism for dense cloud interiors as compared with diffuse media.

In the present paper we attempt to explain the causes of a high polarization-to-extinction ratio for regions with low extinction $\left(A_{v}<1\right)$ as opposed to the low ratio of these parameters for quiescent clouds with high extinction $\left(A_{v} \gg\right.$ 1). Furthermore, we briefly address the challenging problem of why thermal emission polarization (see Hildebrand 1996) observed at far-infrared and submillimeter wavelengths from regions of massive star formation seems to override this tendency.

We address the problem of polarization arising from low extinction and high extinction regions using quantitative models of grain alignment developed recently (Draine \& Weingartner 1996, 1997; Lazarian 1995a, 1995b, 1995c, 1995d; Lazarian \& Draine 1997a; Lazarian \& Roberge 1997a, 1997b; Roberge, Hanany, \& Messinger 1995). In particular, we apply these theoretical results to the filamentary dark cloud L1755 in Ophiuchus.

In what follows we first provide the data about L1755, which we select as a representative dark filamentary cloud $(\S 2)$, analyze the dynamics of dust grains ( $(3)$, and then calculate the measures of grain alignment in L1755 predicted by different mechanisms ( $(4)$. The implications of our results for other dense clouds are given in $\S 5$.

\section{L1755 DARK CLOUD}

We select this cloud for detailed study because of the abundance of information about it available in the literature (see Table 2). The extensive studies of ${ }^{13} \mathrm{CO}$ in Loren (1989a, 1989b) provide us with the density and velocity information, the Zeeman studies in Goodman \& Heiles 


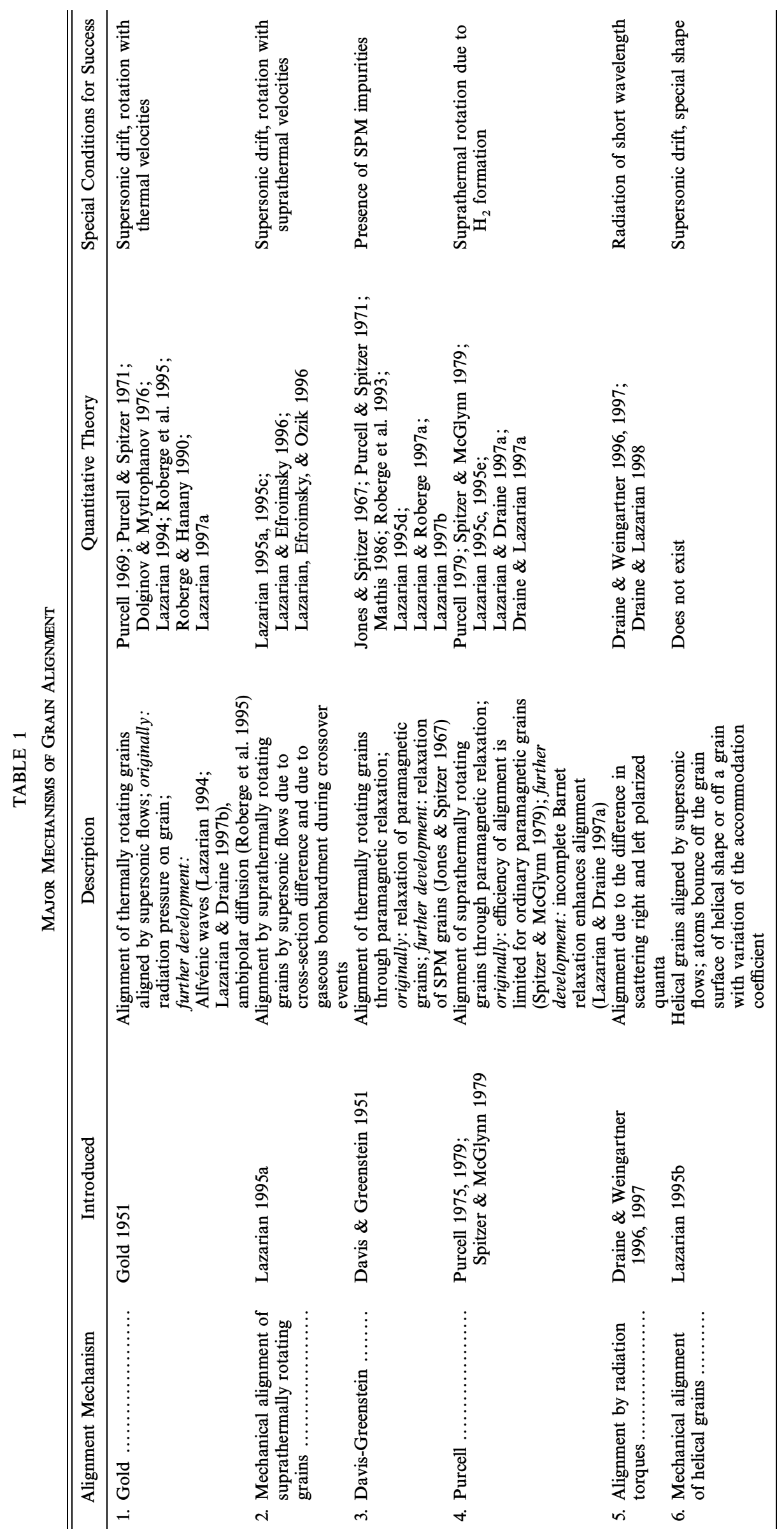


TABLE 2

Physical Quantities Relevant to Models of Grain Alignment IN L1755 IN OPHIUCHUS

\begin{tabular}{cccc}
\hline \hline Quantity & $r<0.05 \mathrm{pc}$ & $0.05 \leq r<0.15 \mathrm{pc}$ & $0.15<r \leq 0.5 \mathrm{pc}$ \\
\hline$T_{\text {gas }}(\mathrm{K}) \ldots \ldots \ldots \ldots$ & 10 & 12 & 18 \\
$T_{d}(\mathrm{~K}) \ldots \ldots \ldots \ldots$ & 12 & 20 & 20 \\
$n_{\text {gas }}\left(\mathrm{cm}^{-3}\right) \ldots \ldots$ & $3 \times 10^{4}$ & $3 \times 10^{3}$ & $3 \times 10^{2}$ \\
$B(\mu \mathrm{G}) \ldots \ldots \ldots \ldots$ & 30 & 20 & 10 \\
$\sigma_{v}\left(\mathrm{~km} \mathrm{~s} \mathrm{~s}^{-1}\right) \ldots \ldots$ & 0.2 & 0.4 & 0.6 \\
$x_{e} \ldots \ldots \ldots \ldots \ldots$. & $2 \times 10^{-7}$ & $6 \times 10^{-6}$ & $1.3 \times 10^{-4}$ \\
$n_{\mathrm{H}} / n_{\text {gas }} \ldots \ldots \ldots \ldots$ & $\sim 3 \times 10^{-3}$ & $\sim 10^{-2}$ & $\sim 10^{-1}$ \\
\hline
\end{tabular}

Notes.-We used data from Benson \& Myers 1989; Bergin, Langer, \& Goldsmith 1995; de Geus, Bronfman, \& Thaddeus 1990; Goodman et al. 1995; Goodman \& Heiles 1994; Loren 1989a, 1989b; McKee 1989; Myers \& Khersonsky 1995; Wood, Myers, \& Daugherty 1994; van Dishoeck et al. 1993. The gas temperature $T_{\text {gas }}$ is taken from $\mathrm{NH}_{3}$ and $\mathrm{CO}(12)$ lines; each is uncertain by $\sim 2 \mathrm{~K}$. For the inner region, $T_{\text {gas }}$ approaches the dust temperature $T_{d}$, which corresponds to the notion of thermal coupling of gas and grains in molecular clouds. The value of $T_{d}$ for outer regions follows from estimates for a standard radiation field; see Draine 1978. The values of gas density $n_{\text {gas }}$ are estimated from $\mathrm{NH}_{3}$ and $\mathrm{CO}_{(13)}$ lines and the requirement that $A_{v}$ increases toward the cloud center. The estimate for magnetic field $B$ follows from the requirement that it lies between (1) the "background" measured in the nearby regions in H I Zeeman (Goodman \& Heiles 1994) and (2) the "equipartition" field strength (Myers \& Khersonsky 1995). The estimate of the velocity dispersion $\sigma_{v}$ is based on $\sigma_{v}=$ FWHM of line profile $=(8 \ln 2)^{1 / 2} \sigma_{v}$ for $\mathrm{NH}_{3}$ and ${ }^{13} \mathrm{CO}$ lines. The ionization ratio $x_{e}=n_{e} / n_{\text {gas }}$ is based on a model of photoionization and cosmicray ionization for a clumpy cloud; see Myers \& Khersonsky 1995.

(1994) provide us with estimates of magnetic field intensity, and optical and near-infrared polarization data are available in Goodman et al. (1990) and in Goodman et al. (1995), respectively. An additional advantage is that the extinction of L1755 changes from $A_{v} \approx 1$ at the edge to $A_{v} \approx 10$ at the center, which enables us to study the substantial variations of polarization with extinction within the same cloud. The concentration of atomic hydrogen is taken from the models of van Dishoeck et al. (1993) and Bergin, Langer, \& Goldsmith (1995). Fractional ionization is estimated using the results of Myers \& Khersonsky (1995). The physical parameters in the outermost layers of L1755 are similar to the neutral diffuse interstellar medium (ISM), and they match the parameters of the dark clouds as density increases (see Table 2). In what follows we will call the part of L1755 within the cylindrical radius $r_{1} \approx 0.05 \mathrm{pc}$ the "inner region," the next shell up to $r_{2} \approx 0.15 \mathrm{pc}$ the "intermediate region," and the regions up to $r_{3} \approx 0.5 \mathrm{pc}$ the "outer region." Such a division involves a degree of arbitrariness, but it suits the purpose of obtaining crude estimates. The sketch of our model of L1755 is presented in Figure 1.

In the interior region, the gas density is high $\left(\sim 10^{4}\right.$ $\mathrm{cm}^{-3}$; see Benson \& Myers 1989), and the magnetic field intensity is $\approx 30 \mu \mathrm{G}$. This estimate of field strength corresponds to the requirement that it lies between values measured in nearby regions by $\mathrm{H}$ I Zeeman observations (Goodman \& Heiles 1994) and the estimates of the field obtained through equipartition arguments (Myers \& Khersonsky 1995). For the outer region of the cloud, we assume that the field strength decreases to match the mean interstellar value of $\sim 3 \mu \mathrm{G}$ (Myers 1987).

The simultaneous change of field strength and gaseous density influences the velocity with which disturbances can propagate in these magnetized regions. Assuming that the line broadening is caused by Alfvénic turbulence (Arons \& Max 1975), we can expect that the line width should decrease toward the higher density regions of L1755, and
${ }^{13} \mathrm{CO}$ Emission

Model Cloud

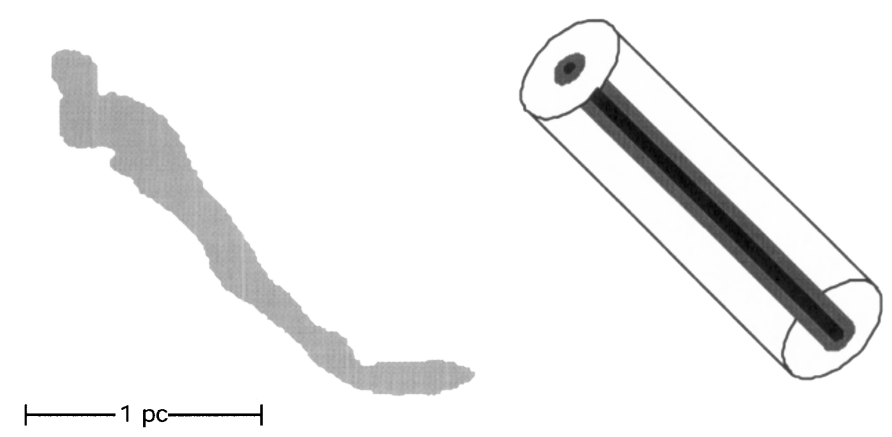

FIG. 1.-Region with intensity greater than $3 \mathrm{~K} J=1-0$ line of ${ }^{13} \mathrm{CO}$ in L1755 (Loren 1989a, 1989b) and the model adopted. The interior region has radius (darkest area) $r_{1}=0.05 \mathrm{pc}$, the intermediate region has radius $r_{2}=0.15 \mathrm{pc}$, and the outer region has radius $r_{3}=0.5 \mathrm{pc}$.

this decrease is, in fact, observed. The ionization ratio should increase outward due to cosmic-ray ionization as the density decreases, and photoionization increases as the extinction decreases (Myers \& Khersonsky 1995).

\section{GRAIN ROTATION}

Before addressing the problem of alignment, we should discuss the processes that determine grain rotation, since the nature of the rotation is an important factor in the alignment.

Thermal rotation.-For thermal rotation it is easy to find the angular velocity of grain rotation, $\omega_{T} \approx$ $\left(k T_{\text {gas }}\right)^{1 / 2} /\left(\rho a^{5}\right)^{1 / 2}$, where $a$ and $\rho$ are, respectively, the effective "radius" of a grain and its density, while $T_{\text {gas }}$ is the gas temperature. Thermal rotation is also referred as "Brownian rotation."

Suprathermal rotation.-It was shown by Purcell (1979) that $\mathrm{H}_{2}$ formation on grain surfaces spins grains up to much larger "suprathermal" angular velocities. Indeed, it is widely believed that the formation of molecular hydrogen mostly takes place at particular catalytic sites, which are frequently called active sites. From these sites, $\mathrm{H}_{2}$ molecules are being ejected with high velocities, and as a result the sites act as tiny rocket engines attached to a grain. These "Purcell rockets" distributed over the grain surface with a mean distance of $l$ between them can accelerate interstellar grains up to the high angular velocity $\omega_{\mathrm{H}_{2}}$ (see Table 3 ).

We assume (see Lazarian 1995c; Roberge et al. 1995) that in dark clouds the ratio is $\omega_{\mathrm{H}_{2}} / \omega_{T} \approx 1$. The rotation of such grains is analogous to the Brownian motion, although the analogy is not exact (Lazarian \& Draine 1997a).

Rotation arising from radiative torques.-Irregular grains can be expected to have nonzero helicity, and these "helical" grains scatter left- and right-circularly polarized light differently. Like the Purcell mechanism, this causes grain spin-up (Dolginov \& Mytrophanov 1976; Lazarian 1995c). ${ }^{6}$ A comprehensive study of the effect of these radiative torques has started only recently (Draine 1996; Draine \& Weingartner 1996, 1997). This study has revealed that under some conditions radiative torques can be stronger than "Purcell rocket" torques. Although a full theory of the process has not yet been completed, ${ }^{7}$ the encouraging

${ }^{6}$ Although both scattering of radiation and $\mathrm{H}_{2}$ formation coerce grains to rotate, we discuss these causes separately, as the anisotropy of the radiation field introduces new effects that we discuss in $\S 4$. 
TABLE 3

Rotational Velocities of Grains in Diffuse Interstellar Media, Dark Clouds, and REgions of MASSIVE Star Formation

\begin{tabular}{cccc}
\hline \hline Parameters & Outer Region of L1755 & Dark Cloud Interiors & Cloud-forming Massive Stars \\
\hline$T_{\text {gas }}(\mathrm{K}) \ldots \ldots \ldots \ldots$ & 18 & 10 & 45 \\
$T_{d}(\mathrm{~K}) \ldots \ldots \ldots \ldots$ & 20 & 12 & 45 \\
$n_{\mathrm{H}}\left(\mathrm{cm}^{-3}\right) \ldots \ldots \ldots$ & 30 & 10 & $10^{3}$ \\
$u_{\text {rad }}\left(u_{\text {ISRF }}\right) \ldots \ldots$ & 1 & 0.07 & 240 \\
$n_{\text {gas }}\left(\mathrm{cm}^{-3}\right) \ldots \ldots$ & $3 \times 10^{2}$ & $3 \times 10^{4}$ & $10^{5}$ \\
\hline
\end{tabular}

\begin{tabular}{|c|c|c|c|c|c|c|c|c|c|}
\hline \multirow[b]{2}{*}{$a(\mu \mathrm{m})$} & \multicolumn{3}{|c|}{ OUtER REGION OF L1755 } & \multicolumn{3}{|c|}{ DARK Cloud INTERIORS } & \multicolumn{3}{|c|}{ Cloud-forming Massive Stars } \\
\hline & $\omega_{T}, \mathrm{~s}^{-1}$ & $\omega_{\mathrm{rad}}^{2} / \omega_{T}^{2}$ & $\omega_{\mathrm{H}_{2}}^{2} / \omega_{T}^{2}$ & $\omega_{T}, \mathrm{~s}^{-1}$ & $\omega_{\mathrm{rad}}^{2} / \omega_{T}^{2}$ & $\omega_{\mathbf{H}_{2}}^{2} / \omega_{T}^{2}$ & $\omega_{T}, \mathrm{~s}^{-1}$ & $\omega_{\mathrm{rad}}^{2} / \omega_{T}^{2}$ & $\omega_{\mathrm{H}_{2}}^{2} / \omega_{T}^{2}$ \\
\hline 0.02 . & $3.1 \times 10^{6}$ & $1.2 \times 10^{-5}$ & $8.4 \times 10^{2}$ & $2.2 \times 10^{6}$ & $1.8 \times 10^{-6}$ & $\mathrm{U}$ & $4.7 \times 10^{6}$ & $1.4 \times 10^{-2}$ & $\mathrm{U}$ \\
\hline $0.05 \ldots \ldots$ & $3.2 \times 10^{5}$ & $2.4 \times 10^{-2}$ & 36 & $2.3 \times 10^{5}$ & $7.0 \times 10^{-5}$ & $\mathrm{U}$ & $4.7 \times 10^{5}$ & 1.3 & $\mathrm{U}$ \\
\hline $0.2 \ldots \ldots$ & $9.9 \times 10^{3}$ & 22 & 158 & $7.0 \times 10^{3}$ & 0.1 & $\mathrm{U}$ & $1.5 \times 10^{4}$ & $1.4 \times 10^{3}$ & $\mathrm{U}$ \\
\hline
\end{tabular}

NoTES. - $\omega_{\text {rad }}$ taken from Draine $\&$ Weingartner 1996 does not account for the starlight reddening (the latter decreases radiative torques). We assumed the accommodation coefficient $\gamma$ equals $0.1 . \mathrm{U}=$ "uncertain" and reflects the existing theoretical ambiguity in determining the number of active sites when the photodesorption is low. $u_{\mathrm{ISRF}} \approx 8.6 \times 10^{-13} \mathrm{ergs} \mathrm{cm}^{-3}$ corresponds to the energy of the averaged interstellar radiation field; see Draine 1978. The choice of grain sizes is influenced by Kim \& Martin 1994, 1995a, 1995b results.

results so far can be treated as preliminary evidence that grains of irregular shape achieve substantial suprathermal rotation due to radiative torques. In Table 3 we reproduce data from numerical runs in Draine \& Weingartner (1996) to show that the effects of the radiative torques are substantial both in outer regions of L1755 and in regions of massive star formation. Whether Purcell torques or radiative torques dominate in the outer regions of L1755 is not evident from these data.

\section{MECHANISMS OF ALIGNMENT}

\subsection{Davis-Greenstein Alignment}

"Davis-Greenstein alignment" is the paramagnetic alignment of thermally rotating grains (see Table 1). A grain whose angular velocity has a nonzero component $\Omega_{\perp}$ perpendicular to the direction of the interstellar magnetic field $\boldsymbol{B}$ experiences alternating magnetization. This causes dissipation and a corresponding decrease in $\Omega_{\perp}$. If the scale for paramagnetic dissipation, $t_{r}$, is much less than the timescale over which gaseous bombardment restores $\Omega_{\perp}$ (the latter coincides with the gas damping time, $t_{d}$ ), grains should be well-aligned in the diffuse ISM. We stress that this condition may not be satisfied in dense clouds, as the efficiency of paramagnetic relaxation also depends on the gas-grain temperature ratio (Jones \& Spitzer 1967), and this ratio is close to unity for dense gas (see Table 2). Davis-Greenstein alignment also depends on the magnetic properties of interstellar grains (see the review by Draine 1996), so we need to distinguish the alignment of ordinary paramagnetic grains and the alignment of superparamagnetic (SPM) and superferromagnetic grains. For the sake of simplicity, in what follows we will refer to both cases of "super"-grains as "SPM grains."

Theoretical studies of Davis-Greenstein alignment show that this mechanism predicts less interstellar polarization than that observed if grains have properties of ordinary

\footnotetext{
${ }^{7}$ Dolginov \& Mytrophanov (1976) attempted to calculate the radiative torques in the Rayleigh-Hans approximation. However, their predictions were not confirmed by recent numerical runs in Draine \& Lazarian (1997b). Neither of the studies so far addresses an important issue of crossovers.
}

paramagnetic materials (see Mathis 1986; Martin 1995). ${ }^{8}$ Therefore, in our study of Davis-Greenstein relaxation, we assume that interstellar grains are SPM (see experimental evidence in Goodman \& Whittet 1995). SPM grains align on a timescale that is very small in comparison to the gaseous damping time (the ratio of the two can be as small as $10^{-5}$ ). As a result, randomization through gas-grain collisions becomes negligible on the timescale of grain alignment, and the measure of alignment becomes limited only by the gas-grain temperature ratio.

According to Table $2 T_{d} / T_{\text {gas }}$ is greater than unity for all three regions of L1755. For these circumstances, Figure 2 presents a plot of the Rayleigh reduction factor (Greenberg 1968) for thermally spinning SPM grains:

$$
R=\frac{3}{2}\left\langle\cos ^{2} \beta-\frac{1}{3}\right\rangle,
$$

where $\beta$ is the angle between the axis of symmetry of an oblate spheroid ${ }^{9}$ and the ambient magnetic field, while here and further on angle brackets $\langle\cdots\rangle$ denote ensemble averaging. This plot accounts for both incomplete coupling of $\boldsymbol{J}$ with the grain principal axis of maximal inertia (henceforth the axis of major inertia) and the partial alignment of $\boldsymbol{J}$ with respect to the external magnetic field (see Lazarian 1995c). We adopt this $R$ as our measure of alignment.

In general, $R$ ranges from -0.5 , corresponding to perfect alignment with grains longest axis parallel to the magnetic field direction, to +1 , corresponding to perfect alignment with longer grain axis perpendicular to the magnetic field. $R=0$ corresponds to "no alignment." Negative values of $R$ in Table 4 and in Figure 2 indicate that grains tend to align with their long axes parallel to the external magnetic field; this is a consequence of the fact that $T_{d}>T_{\text {gas }}$ (Jones \& Spitzer 1967). We will call the alignment with negative $R$

\footnotetext{
${ }^{8}$ Very small grains may still be aligned, as the paramagnetic relaxation efficiency increases with the decrease of grain size more quickly compared to the efficiency of randomization. Therefore, small grains may trace magnetic field intensity; the corresponding paper that relates this quantity with the degree of UV polarization is under preparation (Lazarian \& Martin 1997).

9 There are indications that aligned grains in molecular clouds are oblate rather than prolate (Hildebrand 1988).
} 


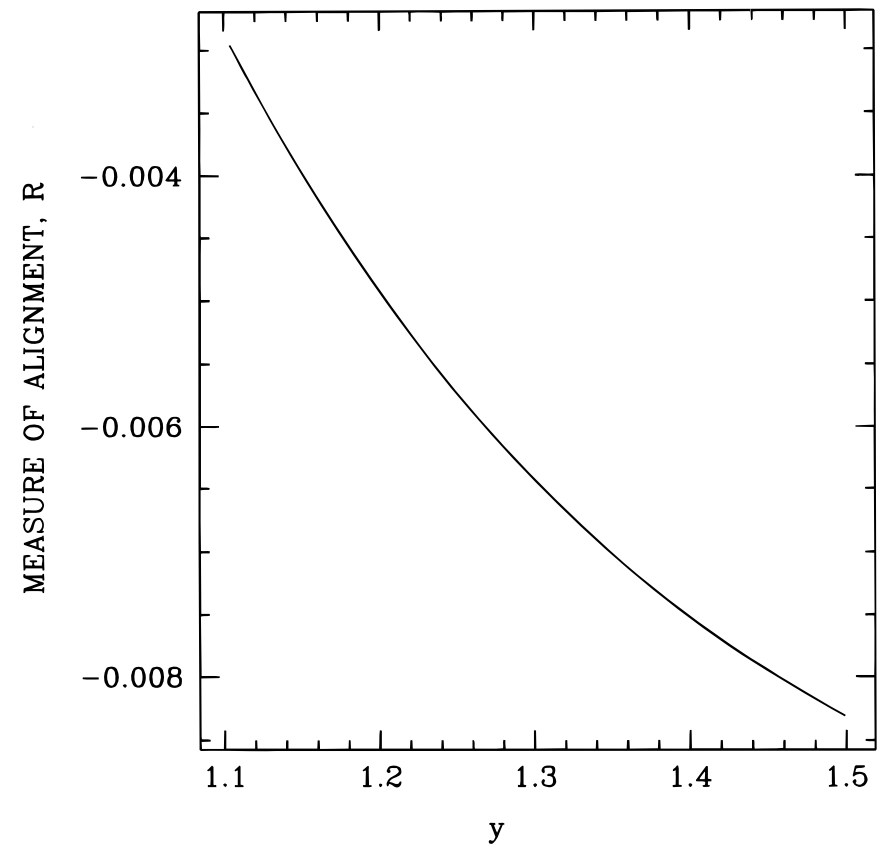

Fig. 2.-The measure of Davis-Greenstein alignment for superparamagnetic grains as a function of $y=T_{d} / T_{m}, T_{m}=\left(T_{\text {gas }}+T_{d}\right) / 2$ for the grains with the ratio of moments of inertia $I_{z} / I_{\perp}=8 / 5$. The negative measures of alignment correspond to alignment with long axes preferentially directed along magnetic field lines. This is the consequence of $T_{d}$ being greater than $T_{\text {gas }}$. "wrong alignment" to distinguish it from the "right alignment" with $R>0$ that dominates at least in the diffuse interstellar medium. The very small absolute values of $R$ listed in Table 4 under "paramagnetic alignment" for every case involving thermal grain rotation show that DavisGreenstein alignment of even SPM grains is virtually ineffective throughout L1755.

In specific cases we can relate $R$ to observed polarization. For instance, the intensity of the polarized component of starlight passing through a cloud is proportional to the difference in the maximal and minimal extinction cross sections for an ensemble of aligned nonspherical grains. If the extinction cross section for radiation with electric vector $\boldsymbol{E}$ parallel to the symmetry axis of an oblate grain is $C_{\|}$and the cross section for $E$ perpendicular to this axis is $C_{\perp}$, then the polarized radiation intensity is proportional to $R\left(C_{\perp}-C_{\|}\right) \cos \xi$, where $\xi$ is the angle that the magnetic field makes with the direction of observation (see Roberge 1996). The difference $\left(C_{\perp}-C_{\|}\right)$depends on the optical properties, oblateness, and sizes of grains (see Draine \& Lee 1984). Even for perfect alignment of highly polarizing grains, polarization arising from dichroic extinction does not exceed a few percent. To account for the observed polarization of $\sim 1 \%, R>0.1$ is usually required (see Martin 1978; Whittet 1992).

No appreciable Davis-Greenstein alignment is expected anywhere in L1755 where gas and dust temperatures are

TABLE 4

Predicted Grain Alignment Measure R in Regions of L1755

\begin{tabular}{|c|c|c|c|c|}
\hline \multirow[b]{2}{*}{ ALIGNMENT } & \multirow[b]{2}{*}{ MECHANICAL } & \multicolumn{2}{|c|}{ PARAMETRIC } & \multirow[b]{2}{*}{ RADIATIVE TORQUES } \\
\hline & & Ordinary & Inclusions & \\
\hline \multicolumn{5}{|c|}{$\begin{array}{c}\text { Outer Region } \\
A_{v}<0.9 \text { mag, } 0.15<r<0.5 \mathrm{pc}\end{array}$} \\
\hline Thermal............ & $\begin{array}{c}0.08 \\
(1)\end{array}$ & $-9 \times 10^{-6}$ & $-1.6 \times 10^{-3}$ & N.A. \\
\hline Suprathermal...... & $\begin{array}{c}0.22 \\
(2)\end{array}$ & $\begin{array}{l}0.7 \\
(4)\end{array}$ & $\begin{array}{l}1^{*} \\
(4)\end{array}$ & $\begin{array}{l}\sim 1^{*} \\
(5)\end{array}$ \\
\hline \multicolumn{5}{|c|}{$\begin{array}{c}\text { Intermediate Region } \\
A_{v} \approx 3 \mathrm{mag}, 0.05<r<0.15 \mathrm{pc}\end{array}$} \\
\hline Thermal............ & $\begin{array}{c}0.08 \\
(1)\end{array}$ & $-2.4 \times 10^{-5}$ & $-5.7 \times 10^{-3}$ & N.A. \\
\hline Suprathermal...... & $\begin{array}{l}0.1^{*} \\
(2)\end{array}$ & $\begin{array}{l}7 \times 10^{-5} \\
(4)\end{array}$ & $\begin{array}{l}0.1^{*} \\
(4)\end{array}$ & $\begin{array}{l}\sim 0 \\
(5)\end{array}$ \\
\hline \multicolumn{5}{|c|}{$\begin{array}{c}\text { Inner Region } \\
A_{v}>3 \mathrm{mag}, r<0.05 \mathrm{pc}\end{array}$} \\
\hline Thermal............ & $\begin{array}{c}0 \\
0 \\
(1)\end{array}$ & $-1.7 \times 10^{-5}$ & $-3 \times 10^{-3}$ & N.A. \\
\hline Suprathermal...... & $\begin{array}{l}0 \\
0 \\
(2)\end{array}$ & $\begin{array}{l}6 \times 10^{-5} \\
(4)\end{array}$ & $\begin{array}{c}0.01^{*} \\
(4)\end{array}$ & $\begin{array}{c}0 \\
(5)\end{array}$ \\
\hline
\end{tabular}

Notes. - The grain axis ratio adopted is $\frac{2}{3}$, and the grain radius is $10^{-5} \mathrm{~cm}$. N.A. $=$ not applicable. The calculations are made using results from Draine \& Weingartner 1996; Lazarian 1994, 1995c, 1996; Lazarian \& Draine 1997a; Lazarian \& Roberge 1997a. The number for $R$ arising from radiative torques is still uncertain, and $R \approx 1$ is our educated guess. The numbers in parentheses correspond to the number of the mechanism in Table 1 . The estimates for nonthermal alignment in the intermediate region are obtained assuming that $\omega_{\mathrm{H}_{2}} / \omega_{T} \sim 1$ (see Table 3 ). In this case $R$ is positive, as the effective temperature of grain rotation is greater than the temperature of grain material. Asterisks indicate the measures of alignment that dominate. For the inner region, in the presence of supersonic ambipolar diffusion, the measures of mechanical alignment would coincide with the corresponding column of the intermediate region panel. 
nearly equal. This conclusion is a fortiori true if grains are not superparamagnetic (see Table 4).

\subsection{Purcell Alignment}

Purcell alignment is the paramagnetic alignment of grains rotating suprathermally. Such grains are not susceptible to disorientation arising from gaseous bombardment. As a result, Purcell alignment does not depend on the gasgrain temperature ratio, and even if they are not superparamagnetic, grains can be efficiently aligned in the typical interstellar magnetic field (Lazarian \& Draine 1997a, 1997b; Draine \& Lazarian 1997a, 1997b) (see Table 1).

As shown above, the inefficiency of Davis-Greenstein alignment of SPM grains in L1755 stems from the insignificant difference in grain-gas temperatures. However, it was pointed out in $\S 3$ that grain rotation can be substantially faster than the gas kinetic temperature and that "Purcell rockets" may be a major cause of suprathermal rotation. For these rockets to work, a supply of "fuel" is required. This fuel is atomic hydrogen in the ambient gas. As the concentration of atomic hydrogen decreases toward the inner regions of L1755, grain rotation slows down (see Table 3). However, in the outer regions of L1755, the concentration of atomic hydrogen approaches that of diffuse regions, and the rotation is suprathermal (see Table 3). Therefore, according to the results of Spitzer \& McGlynn (1979; see also Table 4), those grains that are SPM should be perfectly aligned in the outer regions of L 1755 .

A different question is the alignment of suprathermally rotating grains of ordinary paramagnetic substance. Until recently, it was believed that an appreciable Purcell alignment could be achieved only under a rather restrictive condition of the long-lived spin-up (Spitzer \& McGlynn 1979; Lazarian 1995b). However, a study by Lazarian \& Draine (1997a) has revealed that a substantial alignment is attainable even for short-lived spin-ups. This alignment arises from a high degree of correlation between grain angular momentum $\boldsymbol{J}$ before and after a crossover. This correlation was missed by earlier research that assumed that during spin-ups $\boldsymbol{J}$ is perfectly aligned with the grain axis of major inertia (see Spitzer \& McGlynn 1979). Recent study of this internal alignment (Lazarian \& Roberge 1997a) has shown that coupling of $\boldsymbol{J}$ with the major inertia axis is valid up to thermal fluctuations within the grain material. These fluctuations, contrary to intuitive expectations, enhance the alignment. Our estimates that account for thermal fluctuations (see Table 4) indicate that the Purcell mechanism can provide notable alignment of ordinary paramagnetic grains (i.e., $R \approx 0.7$ ) in the outer region of L1755 (see Table 4).

For intermediate regions in Figure 1 where $\omega_{\mathrm{H}_{2}}<\omega_{T}$, the effective grain rotational temperature will be at most twice that of the ambient gas. Such rotation is only marginally suprathermal, and disorientation during spin-ups is substantial. Even under the most favorable conditions, $R$ is still less than 0.1 in the intermediate region.

The Purcell alignment is negligible for the inner region of L1755 (see Table 4).

\subsection{Radiative Torques}

As was pointed out in $\S 2$, radiative torques are different from those arising from $\mathrm{H}_{2}$ formation. Unlike the isotropic bath of $\mathrm{H}$ atoms that provide the "fuel" for Purcell's spinup, the photons needed to drive radiative alignment are often anisotropically distributed in space. This anisotropy results in a peculiar mechanism of grain alignment (see mechanism 5 in Table 1), which tends to align the short axis of the grain with the magnetic field (i.e., "right alignment").

The matter of radiative torques is currently an issue of intense theoretical research (Draine \& Lazarian 1997a, 1997b), and a number of questions need to be answered before quantitative conclusions can be drawn. Therefore, some degree of uncertainty is present in our discussion here. Our conclusions here are based on the results in Draine \& Weingartner (1996, 1997). A study in Draine \& Lazarian (1997a, 1997b) has shown that for generally accepted values of active sites' density and $\mathrm{H}_{2}$ formation efficiency, radiative torques only marginally alter the dynamics that is dominated by $\mathrm{H}_{2}$ torques. However, in view of the huge uncertainties involved, it is also possible that alignment by radiative torques may be the dominant mechanism of alignment. We hope that future theoretical work in the field will provide us with detailed predictions that can be used for testing the theory against observations.

Table 4 shows that for both the intermediate and inner regions of L1755, the radiative torques are too weak to produce any measurable grain alignment. At the same time, these torques can be important in the outer regions of L1755.

\subsection{Mechanical Alignment}

Both thermally and suprathermally rotating grains can be aligned because of collisions with a gaseous flow. The mechanical alignment of thermally rotating grains was described by Gold (1951), while the mechanical alignment of suprathermally rotating grains was described only recently (Lazarian 1995a; see Table 1).

The important factor in mechanical alignment is supersonic gas-grain drift. It was pointed out by Lazarian (1994) that Alfvén waves can cause such drift, and this entails alignment with grain long axes perpendicular to magnetic field lines, i.e., the "right alignment." The formal theory of grain drift is given in Lazarian \& Draine (1997b), and alignment measures are calculated in Lazarian (1994, 1995a, 1997a), Lazarian \& Efroimsky (1996), and Lazarian, Efroimsky, \& Ozik (1996).

A spectrum of Alfvénic waves should be present in the interstellar medium, and Alfvénic velocities are greater than sound velocities for most interstellar conditions (Myers 1987). In these circumstances, a crucial test of whether Alfvénic waves cause supersonic drift is to compare the Larmor frequency of grain gyration about magnetic field lines with the cutoff frequency of Alfvénic spectrum (Lazarian 1994; Lazarian \& Draine 1997b). This comparison is difficult because the measured quantities needed to estimate the Larmor frequency are uncertain.

If grains are collisionally charged, then in regions with sufficient ionization, Alfvén waves can cause supersonic grain drift when the grain Larmor frequency is lower than the cutoff frequency for the waves,

$$
\omega_{\max } \approx 1.2 \times 10^{-10}\left(\frac{n_{\text {gas }}}{10^{3}}\right)\left(\frac{x_{e}}{4 \times 10^{-5}}\right) \mathrm{s}^{-1},
$$

where $x_{e}$ is the ionization ratio.

For the outer region of L1755, $\omega_{\max }$ is on the order of $10^{-9} \mathrm{~s}^{-1}$, and the grains there may experience supersonic drift. For grains in the intermediate and inner regions, $\omega_{\max }$ becomes $\sim 6 \times 10^{-12} \mathrm{~s}^{-1}$ and $3 \times 10^{-14} \mathrm{~s}^{-1}$, respectively. 
Therefore, supersonic drift is only marginally possible for the intermediate region and impossible for inner regions of L1755. In Table 4 we provide an estimate for grain alignment in the intermediate region, assuming a collisional charging of the grains. One source of uncertainty in these predictions is a possible helicity of grains (Lazarian 1995b). Unfortunately, a quantitative theory of such an alignment is still to be developed.

If grains are more highly charged through photoemission (Draine 1978) in any region, then the Larmor frequency is much greater than $\omega_{\max }$, and supersonic motion is impossible. This can suppress the alignment by Alfvén waves.

In the presence of supersonic ambipolar diffusion, both thermally and suprathermally rotating grains can be aligned mechanically (see Table 4). However, we do not have evidence for supersonic ambipolar diffusion in L1755. Therefore, we do not discuss this possibility here.

\subsection{Exotic Mechanisms of Alignment}

One mechanism omitted from Table 1 can still be efficient in the interior regions of L1755. This mechanism, suggested by Spitzer \& Tukey (1951), is based on the alignment of magnetic moments of ferromagnetic grains and has been shown to be very inefficient in the diffuse clouds (Davis \& Greenstein 1951). However, its efficiency scales as $B / T_{\text {gas }}$, and therefore if grains are ferromagnetic, it may be active in dark clouds, where the ambient magnetic field $B$ is higher than in diffuse clouds, while the temperature is lower (see Table 2). For a population of exclusively ferromagnetic grains, the measure of alignment is expected to be on the order of few percent (see Lazarian 1995c), but if the abundance of such grains is negligible, this mechanism is of marginal importance for L1755. This mechanism will produce "wrong alignment," with the long grain axis parallel to $\boldsymbol{B}$.

It has been suggested that since shocks can dissociate $\mathrm{H}_{2}$, Purcell alignment might be enhanced in shocked regions of cloud interiors (Johnson 1982). Shocks also cause ambipolar diffusion, which may produce alignment (Roberge \& Hanany 1990; Lazarian 1994; Roberge et al. 1995). The presence of intense sources of cosmic rays or X-rays in the vicinity of a cloud may induce grain alignment. Such radiation can excite grain rotation, enabling Davis-Greenstein alignment of SPM grains (Lazarian \& Roberge 1997b) and/or providing conditions for alignment by Alfvénic waves (Lazarian 1994, 1995a, 1995b, 1995c, 1995d, 1995e; Lazarian \& Draine 1997b). As none of these special conditions are especially applicable to L1755, we conclude that all the known major mechanisms fail in the cloud interior.

\section{DISCUSSION}

\subsection{Joint Action of Several Mechanisms}

We have seen above that all the major mechanisms (see Table 1) fail to produce grain alignment in the interior and intermediate regions of L1755. On the other hand, grain alignment in the outer regions of L1755 is efficient. If, for example, both (1) the Purcell mechanism, providing alignment with measure $R_{1}$, and (2) mechanical alignment by Alfvénic waves, providing the alignment with the measure $R_{2}$, act simultaneously, the overall alignment measure can be calculated using the approach suggested in Lazarian (1996). For suprathermally rotating grains, the formula for the Rayleigh reduction factor is

$$
R \approx \frac{R_{1}+R_{1} R_{2}+R_{2}}{1+2 R_{1} R_{2}}
$$

and this provides $R>0.8$ for the outer region of L1755 in our example. As we pointed out above, the suprathermal rotation of grains arises in outer parts of L1755 as the result of $\mathrm{H}_{2}$ formation on grain surfaces.

The measure of grain alignment versus grain size should be different for radiative torques and superparamagnetic relaxation. This provides a way to distinguish the mechanisms. Another method of separating the mechanisms is to study how alignment varies with environmental conditions. For instance, paramagnetic alignment depends specifically on the concentration of atomic hydrogen, while the alignment caused by radiative torques varies only with extinction. Table 3 indicates that at some optical depth Purcell alignment continues, while the alignment by radiative torques may fail.

Extension of the theories to emission polarimetry.-The results of studies of polarized thermal emission in the submillimeter and far-infrared differ substantially from the studies of background starlight polarization in nearinfrared and optical range (cf. M17 polarization maps in Goodman 1996 and Dotson 1996). The near-infrared background starlight polarimetry indicates that grains are not aligned for $A_{v} \gg 1$, but the far-infrared emission polarimetry reveals aligned grains within clouds of high opacity.

From the point of view of grain alignment theory, this paradox can be explained as a selection effect. Indeed, farinfrared emission polarimetry selects warm grains, often in the vicinity of young massive stars. Such grains find themselves in the environments far from equilibrium. For instance, our Table 3 shows that these grains will rotate suprathermally because of radiative torques. On the contrary, the near-infrared background starlight observations are sensitive to the whole sample of grains along the line from the source to the observer. The situation is aggravated by the fact that observations of background starlight in the optical and near-infrared are most often performed in dark quiescent clouds, while the observations of far-infrared emission are most often performed in the regions of massive star formation (see Davidson et al. 1995; Dotson 1996; Hildebrand et al. 1984, 1990; Hildebrand \& Dragovan 1995). This means that direct comparison of background starlight polarimetry and thermal emission polarimetry is difficult using existing data.

In dark clouds like L1755, we expect to detect polarized emission emanating from the vicinity of any low-mass stars formed there, but the bulk of the cloud interior should not emit much polarized radiation if our understanding of the conditions within L1755 and our theory of grain alignment are adequate.

Magnetic fields from polarization maps.-L1755 is an ordinary filamentary dark cloud, and therefore our conclusions should be applicable to the whole class of similar objects. Thus, we expect near-infrared background starlight polarimetry to reveal the structure of magnetic fields only in dense cloud exteriors and to have no relation to the field structure for the regions corresponding to $A_{v} \gg 1$. To what particular extinction magnetic fields can be traced using background starlight polarimetry remains to be determined (see Arce et al. 1997). This important question should be addressed by concerted efforts of observers and theo- 
reticians. We may further expect that emission polarimetry studies of the regions similar to L1755 may not give insight into the magnetic field structure for these cold highextinction regions, apart from small regions surrounding low-mass stars forming there.

On the contrary, emission studies of regions of massive star formation should be a reliable tool for revealing magnetic field structure there. ${ }^{10}$ This technique, however, is biased against cold regions where dust emission is low.

\section{CONCLUSIONS}

Our quantitative study of six types of alignment mechanisms predicts almost no polarization from the interiors of dark clouds and appreciable polarization from the cloud exteriors. Adopting L1755 as a test case, we have shown that a number of mechanisms, including paramagnetic

\footnotetext{
${ }^{10}$ Although winds and outflows in these regions may cause mechanical alignment with grain long axes parallel to magnetic field lines, results in Lazarian (1997a) shows that such alignment is an exception rather than a rule.
}

alignment of SPM grains and alignment by radiative torques, provide efficient orientation of grains in the outermost regions of this cloud. At some moderate optical depth, still in the outer region, the Purcell alignment of ordinary paramagnetic grains may dominate radiative torques. Because all the alignment mechanisms studied fail in the interior region of L1755, we do not expect significant alignment there. This difference in alignment efficiency can provide a natural explanation of why no increase of polarization with optical depth is seen in L1755 (Goodman et al. 1995), and it is consistent with a prediction of only marginal polarization of thermal emission emanating from grains in the interior of L1755.

To test the picture presented here, it might be useful to measure near-infrared polarization through a dark cloud that contains a cold dense inner zone, as in L1755, and also a localized heat source, such as an embedded cluster.

A. L. gratefully acknowledges the support of NASA grants NAG 5-2773 and NAG 5-2858 at Princeton University and of the Visiting Fellowship at the HarvardSmithsonian Center for Astrophysics.
Arce, H., Goodman, A., Bastien, P., Kenyon, S., Manset, N., \& Sumner, M. 1997, ApJ, in preparation

Arons, J., \& Max, C. 1975, ApJ, 196, L77

Benson, P. J., \& Myers, P. C. 1989, ApJS, 71, 89

Bergin, E. A., Langer, W. D., \& Goldsmith, P. F. 1995, ApJ, 441, 222

Davidson, J. A., Schleuning, D., Dowell, C. D., Dotson, J., \& Hildebrand,

R. H. 1995, in ASP Conf. Ser. 73, From Gas to Stars to Dust, ed.

M. Haas, J. Davidson, \& E. Erickson (San Francisco: ASP), 225

Davis, L., \& Greenstein, J. L. 1951, ApJ, 114, 206

de Geus, E. J., Bronfman, L., \& Thedeus, P. 1990, A\&A, 231, 137

Desch, S. J., \& Roberge, W. G. 1997, ApJ, 475, L115

Dolginov, A. Z., \& Mytrophanov, I. G. 1976, Ap\&SS, 43, 291

Dotson, J. 1996, in ASP Conf. Ser. 97, Polarimetry of the Interstellar Medium, ed. W. G. Roberge \& D. C. B. Whittet (San Francisco: ASP), 290

Draine, B. T. 1978, ApJ, 36, 595

-1996, in ASP Conf. Ser. 97, Polarimetry of the Interstellar Medium, ed. W. G. Roberge \& D. C. B. Whittet (San Francisco: ASP), 16

Draine, B. T., \& Lazarian, A. 1997a, in preparation $1997 \mathrm{~b}$, in preparation

Draine, B. T., \& Weingartner, J. C. 1996, ApJ, 470, 551

1997, ApJ, 480, 633

Gold, T. 1951, Nature, 169, 322

Goodman, A. A. 1995, in ASP Conf. Ser. 73, From Gas to Stars to Dust, ed. J. Davidson, E. Erickson, \& M. Haas (San Francisco: ASP), 45 1996, in ASP Conf. Ser. 97, Polarimetry of the Interstellar Medium, ed. W. G. Roberge \& D. C. B. Whittet (San Francisco: ASP), 325

Goodman, A. A., Bastein, P., Myers, P. C., \& Menard, F. 1990, ApJ, 359, 363

Goodman, A. A., \& Heiles, C. 1994, ApJ, 424, 208

Goodman, A. A., Jones, T. J., Lada, E. A., \& Myers, P. C. 1992, ApJ, 399, 108

. 1995, ApJ, 448, 748

Goodman, A. A., \& Whittet, D. C. B. 1995, ApJ, 455, L181

Greenberg, J. M. 1968, in Nebulae and Interstellar Matter, vol. 7, ed. G. P.

Kuiper \& B. M. Middlehurst (Chicago: Univ. Chicago Press)

Hall, J. S. 1949, Science, 109, 166

Hildebrand, R. H. 1988, QJRAS, 29, 327

-1996, in ASP Conf. Ser. 97, Polarimetry of the Interstellar Medium, ed. W. G. Roberge \& D. C. B. Whittet (San Francisco: ASP), 254

Hildebrand, R. H., \& Dragovan, M. 1995, ApJ, 540, 663

Hildebrand, R. H., Dragovan, M., \& Novak, G. 1984, ApJ, 284, L51

Hildebrand, R. H., Gonatas, D. P., Platt, S. R., Wu, X. D., Davidson, J. A., \& Werner, M. W. 1990, ApJ, 362, 114

Hiltner, W. A. 1949, ApJ, 109, 471

Johnson, P. E. 1982, Nature, 295, 371
CES

Jones, R. V., \& Spitzer, L., Jr. 1967, ApJ, 147, 943

Kim, S.-H., \& Martin, P. G. 1994, ApJ, 431, 783

1995a, ApJ, 442, 172

1995b, ApJ, 444, 293

Lazarian, A. 1994, MNRAS, 268, 713

. 1995a, ApJ, 451, 660

1995b, MNRAS, 277, 1235

1995c, MNRAS, 274, 679

1995d, ApJ, 453, 229

1995e, A\&A, 293, 859

. 1996, in ASP Conf. Ser. 97, Polarimetry of the Interstellar Medium, ed. W. G. Roberge \& D. C. B. Whittet (San Francisco: ASP), 433

1997a, ApJ, 483, 296

1997b, MNRAS, 288, 609

Lazarian, A., \& Draine, B. T. 1997a, ApJ, 487, 248

. 1997b, ApJ, in preparation

Lazarian, A., \& Efroimsky, M. 1996, ApJ, 466, 274

Lazarian, A., Efroimsky, M., \& Ozik, J. 1996, ApJ, 472, 240

Lazarian, A., \& Martin, P. G. 1997, ApJ, in preparation

Lazarian, A., \& Roberge, W. G. 1997a, ApJ, 484, 230

. 1997b, MNRAS, 287, 941

Loren, R. B. 1989a, ApJ, 338, 902

.1989b, ApJ, 338, 925

Martin, P. G. 1978, Cosmic Dust (Oxford: Clarendon)

. 1995, ApJ, 445, L63

Mathis, J. S. 1986, ApJ, 308, 281

McKee, C. F. 1989, ApJ, 345, 78

Myers, P. C. 1987, in Interstellar Processes, ed. D. J. Hollenbach \& H. A. Thronson (Dordrecht: Kluwer), 71

Myers, P. C., \& Khersonsky, V. K. 1995, ApJ, 442, 186

Purcell, E. M. 1969, Physica, 41, 100

. 1975, in Dusty Universe, ed. G. B. Field \& A. G. W. Cameron

(New York: Neal Watson), 155

. 1979, ApJ, 231, 404

Purcell, E. M., \& Spitzer, L., Jr. 1971, ApJ, 167, 31

Roberge, W. G. 1996, in ASP Conf. Ser. 97, Polarimetry of the Interstellar Medium, ed. W. G. Roberge \& D. C. B. Whittet (San Francisco: ASP), 401

Roberge, W. G., DeGraff, T. A., \& Flaherty, J. E. 1993, ApJ, 418, 287

Roberge, W. G., \& Hanany, S. 1990, BAAS, 22, 862

Roberge, W. G., Hanany, S., \& Messinger, D. W. 1995, ApJ, 453, 238

Spitzer, L., Jr., \& McGlynn, T. A. 1979, ApJ, 231, 417

Spitzer, L., Jr., \& Tukey, J. W. 1951, ApJ, 114, 187

van Dishoek, E. F., Blake, G. A., Draine, B. T., \& Lunine, J. I. 1993, in Protostars and Planets III, ed. E. H. Levy \& J. I. Lunine (Tucson: Univ. Arizona Press), 163

Whittet, D. C. B. 1992, Dust in the Galactic Environment (Bristol: IPP)

Wood, D. O. S., Myers, P. C., \& Daugherty, D. A. 1994, ApJS, 95, 457 